\title{
Acesso aos serviços básicos de saúde e fatores associados: estudo de base populacional
}

\author{
Access to basic health services and associated factors: \\ a population-based study
}

Andréia Aparecida De Luca Moore Bonello ${ }^{1}$

Carlos Roberto Silveira Corrêa ${ }^{1}$

${ }^{1}$ Pós-Graduação em Saúde Coletiva, Área de Epidemiologia, Departamento de Saúde Coletiva, Faculdade de Ciências Médicas, Unicamp. R. Tessália Vieira de Camargo 126/ Prédio da Faculdade de Ciência Médicas, Cidade Universitária. 13087-970 Campinas SP Brasil. acbonello@hotmail.com.br

\begin{abstract}
This study sought to identify factors involved in access to the services of a basic health unit. It is a cross-sectional, population-based study involving 101 randomly-selected families residing in the area covered by the health unit. An adult resident of each household was interviewed. The response variable was whether or not the resident frequented the health unit if he/she or anyone in the family required assistance to resolve a health issue. The independent variables investigated were service provision aspects, demographic and socio-economic characteristics, individual habits, morbidities and use of the health unit. In addition to descriptive and univariate analysis, logistic regression was applied in the multivariate analysis. The results show that access to the basic health unit is associated with the treatment received previously $(\mathrm{OR}=3,224)$ with accessibility $(O R=0,146)$ and micro-area of residence $(O R=$ 10,918). These findings suggest that access is related to the impressions created by the care received at the health unit and is based on experiences with the service, but can also be strongly modulated by individual aspects and factors related to the territory.
\end{abstract}

Key words Access, Accessibility, Basic health services
Resumo Este trabalho teve como objetivo identificar os fatores que intervêm no acesso aos serviços de uma unidade básica de saúde. Este é um estudo transversal, de base populacional, que envolveu 101 famílias residentes na área de abrangência da unidade de saúde selecionadas aleatoriamente. Um morador maior de idade de cada residência foi entrevistado. A variável resposta foi o morador ir ou não à unidade de saúde no caso dele, ou de alguém de sua família, precisar de atendimento para resolver um problema de saúde. As variáveis independentes investigadas foram aspectos da oferta de serviços; características demográficas e socioeconômicas; costumes individuais; morbidades e uso da unidade de saúde. Além da análise descritiva e univariada, a regressão logística foi aplicada na análise multivariada. Os resultados mostram que o acesso à unidade básica de saúde está associado com o atendimento recebido anteriormente $(O R=3.224)$, com a naturalidade $(O R=0.146)$ e a microárea de residência $(O R$ $=10.918$ ). Esses achados sugerem que o acesso está relacionado com o imaginário criado sobre o atendimento da unidade de saúde e se baseia nas experiências vivenciadas com o serviço, mas pode também ser fortemente modulado por aspectos individuais e fatores ligados ao território.

Palavras-chave Acesso, Acessibilidade, Serviços Básicos de Saúde 


\section{Introdução}

O conceito de acesso aos serviços de saúde se relaciona: às necessidades de saúde, à demanda, à oferta e ao uso desses serviços.

Andersen $^{1}$ e Penchansky e Thomas ${ }^{2}$, mencionam que o conceito de acesso ainda não está bem definido e que, por isso, ele é empregado de forma imprecisa. Esses autores ${ }^{1,2}$ consideram que a operacionalização do conceito de acesso é complexa e que os métodos para sua avaliação ainda precisam ser melhor elucidados.

Travassos e Martins ${ }^{3}$ analisaram o conceito de acesso formulado por diferentes autores e concluíram que: o entendimento sobre o que é acesso apresenta variações amplas entre eles e que estas são fruto de suas diferentes visões e enfoques sobre o tema. Assim podemos dizer que existe uma dispersão de sentidos no conceito de acesso.

Para Andersen ${ }^{1}$, acesso é um dos elementos dos sistemas de saúde, ligado à organização dos serviços, abrangendo tanto a entrada como o recebimento dos cuidados subsequentes. Para esse autor ${ }^{1}$, a influência do acesso no uso de serviços de saúde é mediada por fatores individuais. Posteriormente, Andersen ${ }^{1}$ ampliou o seu conceito de acesso, incluindo a etapa de utilização de serviços de saúde, passando a considerar que também é influenciado por fatores contextuais (políticas de saúde e oferta de serviços) ${ }^{1}$. Nas últimas revisões do seu conceito, Andersen ${ }^{1}$ procura incluir os efeitos do uso dos serviços na saúde e a satisfação das pessoas com os serviços.

Donabedian ${ }^{4}$ utiliza o termo acessibilidade, o qual define como um dos aspectos da oferta de serviços que interfere na capacidade de os indivíduos fazerem uso dos mesmos e destes responderem às necessidades de saúde de uma determinada população. Este autor ${ }^{4}$ distingue duas dimensões da acessibilidade que se inter-relacionam: a sócio-organizacional e a geográfica.

Para Penchansky e Thomas ${ }^{2}$ o acesso é o reflexo do grau de ajuste entre os clientes e o sistema de saúde, uma relação entre a oferta e os indivíduos. Ideia semelhante à de Donabedian ${ }^{4}$, contudo, diferem desse autor ${ }^{4}$ ao inserirem no conceito atributos dos indivíduos. O conceito de acesso de Penchansky e Thomas ${ }^{2}$ é composto por várias dimensões que expressam a relação entre a oferta e os indivíduos: disponibilidade de serviços; acessibilidade (geográfica); acolhimento; capacidade de compra; e, aceitabilidade.

Para Santos ${ }^{5}$, o acesso representa uma dimensão associada à organização do sistema e dos serviços de saúde importante e imprescindível para que os princípios básicos do Sistema Único de
Saúde (SUS), entre os quais estão a universalidade e a equidade, sejam alcançados.

Diante desses diferentes referenciais, a ideia que prevalece para nós é que o acesso é uma condição necessária para que a população faça uso dos serviços de saúde quando sentir necessidade ${ }^{1-5}$.

Na organização do SUS, o acesso dos usuários deve ocorrer preferencialmente na atenção básica. Contudo, Campos et al. ${ }^{6}$ e Santos ${ }^{5,7}$ mencionam que a atenção básica está longe de constituir a principal porta de entrada no sistema, perdendo espaço para os serviços de média complexidade.

Entendemos que estudar o acesso buscando conhecer quais os fatores que o favorecem e os que o dificultam é importante e necessário para que os serviços básicos de saúde possam organizar e planejar sua atuação.

Assim, este trabalho teve como objetivo identificar os fatores associados ao acesso aos serviços básicos de saúde partindo do pressuposto de que fatores atrelados a diferentes dimensões do indivíduo e dos serviços de saúde se relacionam na sua construção.

\section{Materiais e métodos}

Este foi um estudo quantitativo com desenho transversal, de caráter descritivo e de base populacional, feito com os moradores da área de abrangência da Unidade de Saúde Américo Bertão, Jardim Eldorado, município de Cordeirópolis, SP, Brasil.

Foi selecionada uma amostra aleatória simples, constituída por 100 famílias cadastradas no Sistema de Informação da Atenção Básica (SIAB). O cálculo amostral foi realizado partindo da hipótese de que o fator considerado tinha uma prevalência de $50 \%$, que é a que fornece a amostra de maior tamanho, aceitando-se que aquela a ser encontrada variasse em $10 \%$, com nível de 95\% de confiança (IC95\%), obtendo-se um número igual a 85 famílias, sendo acrescidos $20 \%$, considerando possíveis casos de perdas e recusas durante a coleta, para totalizar uma amostra final próxima a 100 famílias. De cada família foi incluído na pesquisa um morador com 18 anos ou mais para ser entrevistado.

A coleta de dados foi realizada por meio de entrevista, no período compreendido entre 27/06 e 01/07/2011, no horário comercial por uma equipe treinada e calibrada por um dos autores. Se o entrevistador não encontrasse ninguém do domicílio da família sorteada, ele retornaria à casa por mais três vezes, em dias e horários diferentes. Se persistisse o insucesso nas tentativas 
de entrevista, a família selecionada seria substituída pela família subsequente, de acordo com o cadastro do SIAB. No momento da entrevista, os moradores foram informados sobre os objetivos da pesquisa e assinaram o Termo de Consentimento Livre e Esclarecido, autorizado junto ao Comitê de Ética, de acordo com Resolução CNS/ MS 196/96.

As informações foram obtidas por meio de um questionário semiestruturado com questões abertas e fechadas, elaborado conjuntamente pela pesquisadora, pela coordenadora e pelos agentes comunitários de saúde (ACS) do serviço de saúde, levando em consideração o referencial teórico, as características do serviço e as particularidades da população. A fim de validar o instrumento elaborado, foi realizado um estudo piloto em duas etapas, permitindo, desta maneira, fazer ajustes para uma melhor compreensão do entrevistado e do entrevistador antes da realização do estudo.

A variável resposta deste estudo foi a informação do entrevistado ao responder a seguinte pergunta: "Procura a unidade de saúde como primeira opção, quando alguém na família precisa de atendimento de saúde?" A questão permitia as respostas sim e não. Caso a resposta fosse negativa, o entrevistado precisava informar os motivos de não procurar primeiro esse serviço de saúde.

As variáveis preditoras investigadas abrangiam as características da oferta do serviço de saúde que facilitam ou dificultam o seu uso pelos clientes (variáveis geográficas e sócio-organizacionais). Foram também incluídas variáveis demográficas, socioeconômicas, morbidades, composição familiar, uso do serviço de saúde, costumes individuais com influência no acesso e uso dos serviços de saúde.

As variáveis de ordem "geográfica" foram analisadas a partir das seguintes informações: dificuldades para chegar à unidade de saúde (sim e não), se sim quais?; tempo gasto para chegar (trajeto) à unidade de saúde (relato do tempo em minutos); localização da unidade de saúde (classificação e expressão da percepção desse aspecto).

As variáveis "sócio-organizacionais" foram estudadas a partir das seguintes informações: dificuldades para conseguir o agendamento da consulta com o médico ( tempo de espera para ser atendido pelo médico (relato do tempo em dias e expressão da percepção desse tempo); espaço físico, atendimento, rotina de trabalho, horário de funcionamento e profissionais (inclusive o profissional médico) da unidade de saúde (classificação e expressão da percepção desses aspectos).
As variáveis "socioeconômicas" e "demográficas" foram: a data de nascimento (dia/mês/ano); idade (anos completos); sexo (masculino ou feminino); cor (autorreferida); ocupação (Classificação Brasileira de Profissões); escolaridade (anos completos de estudo); problema de saúde grave (sim ou não); se sim quais?; convênio médico ( $\operatorname{sim}$ ou não); se sim, qual?; renda familiar per capita (salários mínimos); disponibilidade de computador no domicilio (sim ou não); disponibilidade de acesso à Internet no domicílio (sim ou não); disponibilidade de automóvel no domicílio ( $\operatorname{sim}$ ou não); microárea de residência $(1,2$, $3,4)$; naturalidade (cidade e estado); tempo de moradia no bairro (dias, meses ou anos).

As variáveis relacionadas à "composição familiar" foram: estado civil (casado, solteiro, amasiado, viúvo, divorciado, separado e outros); número de moradores no domicílio (total de moradores); número de moradores no domicílio que pertencem à família (total de moradores que pertencem à família); número de filhos na família que residem no domicílio (total de filhos).

A variável "uso dos serviços de saúde" foi avaliada mediante a seguinte informação: uso da unidade de saúde, pelo entrevistado ou por alguém da família, nos últimos 6 meses (sim ou não); se sim, quantas vezes e por qual motivo?

As variáveis relacionadas aos "costumes individuais" foram investigadas a partir das às seguintes informações: participação em algum espaço social no bairro (sim ou não); se sim, qual?; participação nas atividades realizadas pelo Posto de Saúde do bairro?" (sim ou não); se não, por qual motivo?; procura de outros serviços para resolver seus problemas de saúde (sim ou não); se sim, quais?

A análise quantitativa dos dados contou com a descritiva, a qual foi realizada por meio do cálculo da média e desvio-padrão para as variáveis numéricas com distribuição normal; da apresentação da mediana e da distribuição de quartis para as variáveis numéricas com distribuição não normal; e por meio de proporções para as variáveis categóricas e lógicas.

Para estudar a associação das variáveis independentes com a variável resposta, algumas variáveis categóricas foram transformadas em variáveis lógicas. A análise univariada foi realizada por meio da razão de Chances, ou do teste qui-quadrado de Pearson $\left(\chi^{2}\right)$, ou do teste exato de Ficher. Apenas as variáveis que estavam associadas com a variável resposta com nível de significância de 5\% foram levadas para o modelo de regressão logística binária, utilizando o procedimento stepwise, sendo adotado o nível de significância de 5\%. 
Todas as análises foram feitas com os programas estatísticos Epi Info e o SAS (Statistical Analysis System).

$\mathrm{Na}$ análise dos dados qualitativos, para cada questão aberta foram criadas categorias a partir da leitura prévia de todo o conteúdo do questionário respondido. Todas as respostas categorizadas foram convertidas em variáveis lógicas. A validação das categorias criadas foi realizada conjuntamente por um dos autores junto a todos os entrevistadores por meio de um processo que buscou, através da leitura de questionários sorteados aleatoriamente e discussões, a classificação das respostas, por todos os envolvidos, de forma idêntica ou semelhante em todos os questionários escolhidos aleatoriamente.

O estudo foi aprovado pelo Comitê de Ética em Pesquisa da Faculdade de Ciências Médicas da Universidade Estadual de Campinas.

\section{Resultados}

No total, foram entrevistados 101 moradores da área de abrangência da unidade de saúde, 77 (76.2\%) deles referiram procurar o serviço de saúde como primeira opção (Tabela 1).

A média de "idade" dos entrevistados é de 41 anos $( \pm 15.6)$ e $74,3 \%$ deles são do sexo feminino, $59.4 \%$ têm a cor autorreferida branca, $75,2 \%$ são naturais de fora do estado de São Paulo; 69.4\% moram no bairro há mais de 10 anos, $25.7 \%$ residem na microárea $1,28.7 \%$ na microárea 2 , $31.7 \%$ na microárea 3 e $13.9 \%$ na microárea 4 , $51.5 \%$ possuem mais de 08 anos completos de estudo; 73,3\% referiram que a renda per capita na sua família era de até um salário mínimo, $37.6 \%$ referiram ter problemas de saúde, $55.4 \%$ não possuem convênio médico, $64.4 \%$ dispõem de computador, $70.3 \%$ não dispõem de internet, $53.5 \%$ possuem automóvel e $28.7 \%$ trabalham como ceramista (Tabela 2).

Tabela 1. Distribuição dos moradores entrevistados segundo a procura da unidade de saúde como primeira opção, quando alguém na família precisa de atendimento de saúde, Jardim Eldorado, Cordeirópolis, 2011.

\begin{tabular}{ll}
\hline \multicolumn{1}{c}{ Variáveis e categorias } & $\mathbf{n}=\mathbf{1 0 1}$ \\
\hline $\begin{array}{l}\text { Procura a unidade de saúde como } \\
\text { primeira opção }\end{array}$ & \\
Sim & $77(76.2 \%)$ \\
Não & $24(23.8 \%)$ \\
\hline
\end{tabular}

Fonte: Questionários de avaliação do acesso 2011.
Em relação às variáveis "composição familiar"; "uso da unidade nos últimos 6 meses" e "costumes individuais", $56.4 \%$ dos moradores entrevistados são casados, $83.2 \%$ referiram ter até cinco moradores no domicílio; $84.2 \%$ até cinco moradores no domicílio que pertencem à família; e $91.0 \%$ até três filhos que residem no domicílio; $89.1 \%$ referiram ter usado a unidade nos últimos 6 meses; $90.1 \%$ não participam das atividades da unidade de saúde; $50.5 \%$ participam de algum espaço social no bairro; e $83.2 \%$ procuram outros serviços para resolver problemas de saúde (Tabela 2).

Em relação às variáveis de ordem "geográfica" e "sócio-organizacional", 96.0\% dos entrevistados não referiram dificuldade para chegar à unidade de saúde; $97.0 \%$ referiram gastar menos de 30 minutos para chegar a ela; $53.5 \%$ referiram dificuldade no agendamento da consulta; $52.5 \%$ relataram ser necessário esperar até 15 dias pela consulta, e $56.4 \%$ percebem este tempo como adequado (Tabelas 2 e 3 ).

Ao analisar a percepção e a classificação dos aspectos "geográficos" e "sócio-organizacionais", a maior parte dos moradores entrevistados percebe de forma adequada e classificou de forma positiva todos os aspectos abordados. Entretanto, um incremento no percentual de entrevistados que percebem a "rotina de trabalho" (42.6\%) de forma inadequada é notado quando comparado ao percentual de entrevistados que percebem da mesma forma os demais aspectos. Da mesma maneira é observado um aumento no percentual de moradores que classificou a "rotina de trabalho" (25.6\%) como regular quando comparado ao percentual de entrevistados que classificou da mesma forma os demais aspectos (Tabela 3).

A análise univariada (Tabela 4) aponta que as variáveis associadas com a variável resposta foram: "microárea de residência" $\left(\mathrm{X}^{2}=7.83 \mathrm{p}=\right.$ 0.04957112), "naturalidade" (OR = 6.40 IC95\% $=2.327-17.61)$, "uso da unidade nos últimos seis meses" $(\mathrm{OR}=4.80-\mathrm{IC} 95 \%=1.316-17.51)$, "participação nas atividades da unidade de saúde" (Teste Fisher $\mathrm{p}=0.0570978$ ), "procura de outros serviços" (Teste Fisher p $=0.0061348$ ), "classificação do atendimento da unidade de saúde" $\left(\mathrm{X}^{2}=\right.$ 20.20 p-valor 0.00114526$)$, "classificação do horário de funcionamento da unidade de saúde" ( $\mathrm{X}^{2}$ $=11.36$ p-valor 0.02281417) e "classificação dos profissionais da unidade de saúde" $\left(\mathrm{X}^{2}=11.29 \mathrm{p}\right.$ valor 0.02352699 ).

No modelo final da regressão logística (Tabela 5) as variáveis que permaneceram foram: "naturalidade" - "ter nascido no Estado de São Paulo" (OR $=0.146$ IC95\% $=0.045-0.476)$, "uso da 
Tabela 2. Distribuição dos moradores entrevistados segundo as variáveis preditoras, Jardim Eldorado, Cordeirópolis, 2011.

\begin{tabular}{|c|c|c|c|}
\hline Variáveis e categorias & $\mathrm{n}=101$ & Variáveis e categorias & $\mathrm{n}=101$ \\
\hline Idade média (dp), anos & 41 anos $( \pm 15.6)$ & Moradores no domicílio & \\
\hline Sexo & & Até cinco & $84(83.2 \%)$ \\
\hline Feminino & $75(74.3 \%)$ & Mais de cinco & $17(16.8 \%)$ \\
\hline Masculino & $26(25.7 \%)$ & Moradores da família no domicílio & \\
\hline Cor (autorreferida) & & Até cinco & $85(84.2 \%)$ \\
\hline Branca & $60(59.4 \%)$ & Mais de cinco & $16(15.8 \%)$ \\
\hline Outras & $41(40.6 \%)$ & Filhos no domicílio & \\
\hline Naturalidade & & Até três & $92(91.0 \%)$ \\
\hline São Paulo & $25(24.8 \%)$ & Mais de três & $09(9.0 \%)$ \\
\hline Outros Estados & $76(75.2 \%)$ & Problema de saúde grave & \\
\hline Tempo moradia bairro & & Sim & $38(37.6 \%)$ \\
\hline Até 10 anos & $31(30.6 \%)$ & Não & $63(62.4 \%)$ \\
\hline Mais de 10 anos & $70(69.4 \%)$ & Convênio médico & \\
\hline Microárea de residência & & $\operatorname{Sim}$ & $45(44.6 \%)$ \\
\hline 1 & $26(25.7 \%)$ & Não & $56(55.4 \%)$ \\
\hline 2 & $29(28.7 \%)$ & Computador & \\
\hline 3 & $32(31.7 \%)$ & Sim & $36(35.6 \%)$ \\
\hline 4 & $14(13.9 \%)$ & Não & $65(64.4 \%)$ \\
\hline Ocupação & & Internet & \\
\hline Ceramista & $29(28.7 \%)$ & Sim & $30(29.7 \%)$ \\
\hline Outras & $72(71.3 \%)$ & Não & $71(70.3 \%)$ \\
\hline Escolaridade & & Automóvel & \\
\hline Até 08 anos & $49(48.5 \%)$ & Sim & $54(53.5 \%)$ \\
\hline Mais de 08 anos & $52(51.5 \%)$ & Não & $47(46.5 \%)$ \\
\hline Renda familiar per capita & & Uso da unidade de saúde nos & \\
\hline Até um SM & $72(73.3 \%)$ & últimos seis meses & \\
\hline Mais de um SM & $29(26.7 \%)$ & Sim & $90(89.1 \%)$ \\
\hline Estado civil & & Não & $11(10.9 \%)$ \\
\hline Casado & $57(56.4 \%)$ & Participação atividades da unidade & \\
\hline Outros & $44(43.6 \%)$ & Sim & $10(9.9 \%)$ \\
\hline Moradores no domicílio & & Não & $91(90.1 \%)$ \\
\hline Até cinco & $84(83.2 \%)$ & Participação espaço social & \\
\hline Mais de cinco & $17(16.8 \%)$ & Sim & $51(50.5 \%)$ \\
\hline Moradores da família no domicílio & & Não & $97(96.0 \%)$ \\
\hline Até cinco & $85(84.2 \%)$ & Procura outros serviços & \\
\hline Mais de cinco & $16(15.8 \%)$ & Sim & $84(83.2 \%)$ \\
\hline Filhos no domicílio & & Não & $17(16.8 \%)$ \\
\hline Até três & $92(91.0 \%)$ & Dificuldade para chegar à unidade & \\
\hline Mais de três & $09(9.0 \%)$ & de saúde & \\
\hline Problema de saúde grave & & Sim & $04(4.0 \%)$ \\
\hline Sim & $38(37.6 \%)$ & Não & $97(96.0 \%)$ \\
\hline Não & $63(62.4 \%)$ & Tempo de trajeto & \\
\hline Convênio médico & & Até 30 minutos & $98(97.0 \%)$ \\
\hline Sim & $45(44.6 \%)$ & Mais de 30 minutos & $03(3.0 \%)$ \\
\hline Não & $56(55.4 \%)$ & Dificuldade agendamento consulta & \\
\hline Computador & & Sim & $54(53.5 \%)$ \\
\hline Sim & $36(35.6 \%)$ & Não & $47(46.5 \%)$ \\
\hline Não & $65(64.4 \%)$ & Tempo de espera & \\
\hline Estado civil & & Até 15 dias & $53(52.5 \%)$ \\
\hline Casado & $57(56.4 \%)$ & Mais de 15 dias & $48(47.5 \%)$ \\
\hline Outros & $44(43.6 \%)$ & & \\
\hline
\end{tabular}

Fonte: Questionários de avaliação do acesso 2011. 
Tabela 3. Distribuição dos moradores entrevistados segundo a percepção e a classificação dos aspectos geográficos e sócio-organizacionais, Jardim Eldorado, Cordeirópolis, 2011.

\begin{tabular}{|c|c|c|c|}
\hline Variáveis e categorias & $\mathrm{n}=101$ & Variáveis e categorias & $\mathrm{n}=101$ \\
\hline Percepção & & Classificação do espaço físico & \\
\hline Percepção do tempo espera & & Ótimo & $04(4.0 \%)$ \\
\hline Adequado & $57(56.4 \%)$ & Bom & $79(78.2 \%)$ \\
\hline Inadequado & $44(43.6 \%)$ & Regular & $15(14.8 \%)$ \\
\hline Percepção da localização & & Ruim & $01(1.0 \%)$ \\
\hline Adequada & $90(89.1 \%)$ & Péssimo & - \\
\hline Inadequada & $11(10.9 \%)$ & Não sabe & $02(2.0 \%)$ \\
\hline Percepção do espaço físico & & Classificação do atendimento & \\
\hline Adequado & $81(80.2 \%)$ & Ótimo & $10(9.9 \%)$ \\
\hline Inadequado & $20(19.8 \%)$ & Bom & $57(56.4 \%)$ \\
\hline Percepção do atendimento & & Regular & $24(23.7 \%)$ \\
\hline Adequado & $62(61.4 \%)$ & Ruim & $02(2.0 \%)$ \\
\hline Inadequado & $39(38.6 \%)$ & Péssimo & $03(3.0 \%)$ \\
\hline Percepção da rotina trabalho & & Não sabe & $05(5.0 \%)$ \\
\hline Adequada & $58(57.4 \%)$ & Classificação do horário de & \\
\hline Inadequada & $43(42.6 \%)$ & funcionamento & \\
\hline Percepção do horário de & & Ótimo & $\begin{array}{c}- \\
72(71) 30()\end{array}$ \\
\hline funcionamento & & Bom & $72(71.3 \%)$ \\
\hline Adequado & $60(59.4 \%)$ & Regular & $23(22.7 \%)$ \\
\hline Inadequado & $41(40.6 \%)$ & Ruim & $04(4.0 \%)$ \\
\hline Percepção dos profissionais & & Péssimo & - \\
\hline Adequado & $72(71.3 \%)$ & Não sabe & $02(2.0 \%)$ \\
\hline Inadequado & $29(28.7 \%)$ & Classificação dos profissionais & \\
\hline Classificação & & Ótimo & $07(7.0 \%)$ \\
\hline Classificação da localização & & Bom & $66(65.3 \%)$ \\
\hline Ótimo & $14(13.8 \%)$ & Regular & $23(22.7 \%)$ \\
\hline Bom & $78(77.3 \%)$ & Ruim & $02(2.0 \%)$ \\
\hline Regular & $06(5.9 \%)$ & Péssimo & - \\
\hline Ruim & $03(3.0 \%)$ & Não sabe & $03(3.0 \%)$ \\
\hline Péssimo & - & & \\
\hline Não sabe & - & & \\
\hline
\end{tabular}

Fonte: Questionários de avaliação do acesso 2011.

unidade de saúde" - "ter usado a unidade" (OR = 5.594 IC95\% = 1.443- 21.695), "microárea de residência” - "residir na microárea 2” (OR = 10.918 IC95\% = 1.495-79.726) e "classificação do atendimento da unidade de saúde" - "ter classificado o atendimento como bom" $(\mathrm{OR}=3.224 \mathrm{IC} 95 \%=$ 1.002-10.378).

\section{Discussão}

O presente estudo traz informações sobre o acesso aos serviços básicos. Os resultados mostram que os fatores associados ao acesso dos moradores ao serviço no qual estão adstritos são: a naturalidade; o uso da unidade de saúde nos últimos seis meses; o local de residência; e a classificação do atendimento da unidade.

Para estudar o acesso, utilizou-se um instrumento elaborado conjuntamente pela pesquisadora, pela coordenadora da unidade de saúde e pelos agentes comunitários, levando-se em consideração o referencial teórico, as características do serviço e as particularidades da população, optando-se por um delineamento de estudo no qual o entendimento de acesso é o de ser uma condição necessária para que o morador faça uso dos serviços de saúde quando sentir necessidade ${ }^{1-5}, \mathrm{e}$ que este uso se dá em função de fatores relacionados tanto com a oferta de serviços ${ }^{4}$, como com os indivíduos ${ }^{1,2}$. Dessa forma, para operacionalizar o conceito de acesso, foi tomado como indicador a 
Tabela 4. Resultados da análise univariada - associação entre procura da unidade de saúde como primeira opção quando alguém na família precisa de atendimento de saúde e as variáveis de interesse do estudo, Jardim Eldorado, Cordeirópolis, 2011.

\begin{tabular}{|c|c|c|c|}
\hline Variáveis & Procura a unidade & $\mathbf{n}$ & Análise univariada $(n=101)$ \\
\hline Sexo & & & $\mathrm{OR}=1.257(0.453-3.491)$ \\
\hline Feminino & 58 & 75 & \\
\hline Cor & & & $\mathrm{OR}=1.06(0.418-2.688)$ \\
\hline Branca & 46 & 60 & \\
\hline Estado civil & & & $\mathrm{OR}=2.193(0.864-5.569)$ \\
\hline Casado & 47 & 57 & \\
\hline Moradores no domicílio & & & $\mathrm{OR}=0.985(0.288-3.362)$ \\
\hline Até cinco & 64 & 84 & \\
\hline Moradores fam. domicílio & & & $\mathrm{OR}=1.083(0.314-3.735)$ \\
\hline Até cinco & 65 & 85 & \\
\hline Filhos no domicílio & & & $\mathrm{OR}=0.375(0.044-3.161)$ \\
\hline Até três & 69 & 92 & \\
\hline Naturalidade/ Estado & & & $\mathrm{OR}=6.402(2.327-17.61)$ \\
\hline Outros Estados & 65 & 76 & \\
\hline Microárea de residência & & & $\mathrm{p}=0.04957112^{\mathrm{x}}$ \\
\hline 01 & 17 & 26 & \\
\hline 02 & 26 & 29 & \\
\hline 03 & 26 & 32 & \\
\hline 04 & 08 & 14 & \\
\hline Tempo de moradia bairro & & & $\mathrm{OR}=1.015(0.405-2.547)$ \\
\hline Até 10 anos & 42 & 55 & \\
\hline Ocupação & & & $\mathrm{OR}=0.75(0.28-2.01)$ \\
\hline Ceramista & 21 & 29 & \\
\hline Escolaridade & & & $\mathrm{OR}=1.437(0.569-3.628)$ \\
\hline Até oito anos & 39 & 49 & \\
\hline Renda familiar per capita & & & $\mathrm{OR}=0.889(0.311-2.543)$ \\
\hline Até um SM & 56 & 74 & \\
\hline Convênio médico & & & $\mathrm{OR}=0.602(0.239-1.513)$ \\
\hline Sim & 32 & 45 & \\
\hline Computador & & & $\mathrm{OR}=0.568(0.223-1.446)$ \\
\hline $\operatorname{Sim}$ & 25 & 36 & \\
\hline Internet & & & $\mathrm{OR}=0.625(0.238-1.643)$ \\
\hline Sim & 21 & 30 & \\
\hline Automóvel & & & $\mathrm{OR}=0.772(0.306-1.95)$ \\
\hline Sim & 40 & 54 & \\
\hline Problema de saúde grave & & & $\mathrm{OR}=2.133(0.762-5.97)$ \\
\hline Sim & 32 & 38 & \\
\hline Uso unidade últimos 6 meses & & & $\mathrm{OR}=4.8(1.316-17.51)$ \\
\hline Sim & 72 & 90 & \\
\hline
\end{tabular}

continua

propensão do morador procurar ou não a unidade de saúde a que está adstrito. Entendemos que na resposta que o morador deu na entrevista estão envolvidas, dentre outras, as dimensões apontadas no modelo conceitual adotado.

Os resultados do estudo mostram que 76.2\% dos moradores entrevistados procurariam a unidade de saúde como primeira opção. Esse dado sugere que a unidade de saúde constitui uma referência para o atendimento de saúde para a maior parte deles, o que indica que a maior parte dos entrevistados tem acesso ao serviço e, por meio deste, ao sistema de saúde. Resultados semelhantes ao do estudo em questão foram encontrados na Pesquisa Nacional por Amostra de Domicílios $(\mathrm{PNAD})^{9}$ e nas investigações realizadas por Corrêa $a^{10}$ e Oliveira et al. ${ }^{11}$.

Os achados deste estudo apontam que a naturalidade; o uso da unidade de saúde; o local de residência; e a classificação do atendimento da 
Tabela 4. continuação

\begin{tabular}{|c|c|c|c|}
\hline Variáveis & Procura a unidade & $\mathbf{n}$ & Análise univariada $(n=101)$ \\
\hline Participação espaço social & & & $\mathrm{OR}=1.026(0.410-2.566)$ \\
\hline Sim & 39 & 51 & \\
\hline Participação ativ. unidade & & & $\mathrm{p}=0.0570978^{\mathrm{y}}$ \\
\hline $\operatorname{Sim}$ & 10 & 10 & \\
\hline Procura outros serviços & & & $\mathrm{p}=0.0061348^{\mathrm{y}}$ \\
\hline Sim & 60 & 84 & \\
\hline Dificuldade chegar unidade & & & $\mathrm{OR}=0.932(0.092-9.404)$ \\
\hline $\operatorname{Sim}$ & 03 & 04 & \\
\hline Tempo de trajeto & & & $\mathrm{OR}=6.909(0.598-79.82)$ \\
\hline Até 30 minutos & 76 & 98 & \\
\hline Dificuldade agendamento & & & $\mathrm{OR}=2.344(0.913-6.015)$ \\
\hline Sim & 45 & 54 & \\
\hline Tempo de espera consulta & & & $\mathrm{OR}=0.585(0.228-1.496)$ \\
\hline Até 15 dias & 38 & 53 & \\
\hline Percepção tempo de espera & & & $\mathrm{OR}=0.445(0.166-1.195)$ \\
\hline Adequado & 40 & 57 & \\
\hline Percepção localização & & & $\mathrm{OR}=1.232(0.3-5.067)$ \\
\hline Adequado & 69 & 90 & \\
\hline Percepção espaço físico & & & $\mathrm{OR}=0.504(0.134-1.895)$ \\
\hline Adequado & 60 & 81 & \\
\hline Percepção atendimento & & & $\mathrm{OR}=1.180(0.420-3.340)$ \\
\hline Adequado & 48 & 62 & \\
\hline Percepção rotina trabalho & & & $\mathrm{OR}=1.19(0.473-2.992)$ \\
\hline Adequado & 45 & 58 & \\
\hline Percepção horário func. & & & $\mathrm{OR}=1.06(0.418-2.688)$ \\
\hline Adequado & 46 & 60 & \\
\hline Percepção dos profissionais & & & $\mathrm{OR}=1.029(0.375-2.825)$ \\
\hline Adequado & 55 & 72 & \\
\hline Classificação localização & & & $\mathrm{p}=0.31300020^{\mathrm{x}}$ \\
\hline Bom & 61 & 78 & \\
\hline Classificação espaço físico & & & $\mathrm{p}=0.08812636^{\mathrm{x}}$ \\
\hline Bom & 60 & 79 & \\
\hline Classificação atendimento & & & $\mathrm{p}=0.00114526^{\mathrm{x}}$ \\
\hline Bom & 48 & 57 & \\
\hline Classificação rot. trabalho & & & $\mathrm{p}=0.23902314^{\mathrm{x}}$ \\
\hline Bom & 48 & 58 & \\
\hline Classificação horário func & & & $\mathrm{p}=0.02281417^{\mathrm{x}}$ \\
\hline Bom & 58 & 72 & \\
\hline Classificação profissionais & & & $\mathrm{p}=0.02352699^{\mathrm{x}}$ \\
\hline Bom & 51 & 66 & \\
\hline
\end{tabular}

Fonte: Questionários de avaliação do acesso 2011. ${ }^{\mathrm{x}} \mathrm{p}$ valor - teste qui-quadrado; ${ }^{\mathrm{y}} \mathrm{p}$ valor - teste exato de Fisher.

Tabela 5. Modelo Final - Regressão Logística - aspectos associados à procura da unidade de saúde como primeira opção, quando alguém na família precisa de atendimento. Jardim Eldorado, Cordeirópolis, 2011.

\begin{tabular}{lcrr}
\hline \multicolumn{1}{c}{ Variável } & Coeficiente & OR & \multicolumn{1}{c}{ IC95\% } \\
\hline Intercepto & 0.0365 & \multicolumn{1}{c}{-} \\
Naturalidade "ter nascido no Estado de São Paulo" & -0.9637 & 0.146 & $0.045-0.476$ \\
Uso da unidade de saúde nos últimos seis meses "S" & 1.0880 & 5.594 & $1.443-21.695$ \\
Microárea "residir na microárea 2" & 1.4699 & 10.918 & $1.495-79.726$ \\
Atendimento "bom" & 0.5854 & 3.224 & $1.002-10.378$
\end{tabular}

Fonte: Regressão Logística - dados questionários de avaliação do acesso 2011. 
unidade são os fatores associados ao acesso dos moradores ao serviço no qual estão adstritos.

A condição "ter nascido no Estado de São Paulo", associa-se negativamente com a "procura da unidade de saúde", sugerindo uma relação entre a história de vida dos moradores com o acesso ao serviço ao qual estão adstritos. Este dado diverge dos encontrados por Halfon et al. ${ }^{12} \mathrm{e}$ Newbold ${ }^{13}$ que, investigando a naturalidade entre outras variáveis, não confirmam a sua associação com o acesso aos serviços de saúde. Da mesma forma, Flores e $\mathrm{Vega}^{14}$, após revisarem as barreiras no acesso aos serviços de saúde, concluíram que a "naturalidade" parece não atuar como uma barreira. A diferença pode ter acontecido pela forma como o dado foi obtido e analisado e pelos contextos diferentes em que os estudos foram realizados. Os resultados do estudo em questão mostram que o acesso está associado com a naturalidade do morador. Essa variável aponta uma característica do morador, cujo significado ainda precisa ser explicado.

A condição "ter usado a unidade de saúde" associa-se positivamente com a "procura da unidade de saúde”. Acesso e uso dos serviços de saúde são dimensões muito próximas, sendo natural e esperado que o fato de o morador ter usado a unidade de saúde aumentasse a sua chance de tornar a procura-la como primeira opção. Vários fatores podem explicar este efeito, como a necessidade de saúde percebida, os aspectos da oferta de serviço, a qualidade e a efetividade do cuidado oferecido, a satisfação do cliente com a assistência recebida, a configuração da unidade de saúde como uma referência para o atendimento e o vínculo criado entre o serviço e os moradores adstritos a ele. Andersen ${ }^{1}$ define acesso a partir do conceito de uso dos serviços de saúde, como exemplo da associação que existe entre o uso da unidade de saúde e o acesso a ela. Para nós, esta associação existe, mas cabe ressaltar que a chance de um morador ter acesso à unidade de saúde está mais associada ao território do que ao uso desta nos últimos seis meses. Ao tentar estabelecer uma correlação entre a "procura da unidade de saúde como primeira opção" e o "uso da unidade de saúde nos últimos seis meses”, observa-se um padrão semelhante: $76.2 \%$ dos moradores entrevistados referiram procurar a unidade; e $89.1 \%$ dos entrevistados referiram ter usado o serviço, percentual $12.9 \%$ maior em relação ao percentual dos entrevistados que referiram procurar a unidade de saúde.

Morar na microárea de residência 2 foi mais um fator que aumentou a chance de o morador procurar a unidade de saúde. Esses resultados apontam que o território interfere no acesso ao serviço de saúde considerado. Para Santos ${ }^{15}$, o território é o espaço do acontecer solidário, que gera uso de diferentes naturezas, as quais pressupõem coexistências e um espaço geográfico ${ }^{15}$. Assim, entendemos que nesse território estão implicados os valores, a cultura e a história dos indivíduos que nele moram, como também os valores desenvolvidos localmente; e que esses fatores desempenham importante influência no acesso que os indivíduos fazem a este serviço. A influência do território também foi encontrada por Kontopantelis et al. ${ }^{16}$, ao estudarem a satisfação do paciente e analisarem a experiência com o acesso aos cuidados primários. Esses ${ }^{16}$ autores atribuem esses achados ao fato de as expectativas individuais do atendimento e a tendência de avaliação do serviço poderem ser modificadas pelo ponto de vista dominante da comunidade local. Travassos et al. ${ }^{17}$, avaliando o padrão de desigualdades geográficas e sociais no acesso aos serviços de saúde, reafirmam que o acesso no Brasil é fortemente influenciado pelo local de residência dos indivíduos.

Outro fator que aumentou a chance de o morador procurar a unidade de saúde foi a de ele ter classificado positivamente o atendimento que recebeu na unidade de saúde. Esse resultado confirma a influência positiva da percepção dos indivíduos no acesso. A influência da percepção que os indivíduos têm em relação aos aspectos da oferta de serviços também foi apontada nos estudos realizados por Barr e Wanat ${ }^{18}$, Garza-Elizondo et al. ${ }^{19}$ e Reed et al. ${ }^{20}$.

Tais autores ${ }^{18-20}$ consideram importante lembrar que a interpretação dada pelos indivíduos é mediada por aspectos sociais, culturais, valores e subjetividade.

Dentre as limitações do estudo, destacam-se o horário em que a coleta de dados foi realizada, o que pode ter comprometido a participação e a obtenção de informações dos moradores que trabalham em jornada integral. Uma pesquisa realizada por Kontopantelis et al. ${ }^{16}$ mostrou que pessoas que trabalham em tempo integral apresentam maior dificuldade no acesso aos serviços básicos de saúde em decorrência da indisponibilidade de atendimento fora do horário normal de trabalho. A estratégia utilizada para a coleta de dados, na qual o entrevistado forneceu as informações pela família, pode ter ocultado ou sobressaído informações. O fato do local de realização do estudo ter envolvido apenas uma unidade de saúde pode ser interpretado como uma dificuldade para a expansão dos seus resultados. Entretanto, entendemos que o rigor no método utilizado permite que os seus achados sejam disseminados. 
Soma-se a isso a configuração do instrumento construído, o que pode ter direcionado as manifestações da população. Contudo, consideramos que o conjunto articulado do conhecimento, da experiência do cotidiano de trabalho nos serviços de saúde e da perspectiva da população trouxe contribuições que valorizaram o significado dos resultados encontrados.

Enfim, os achados sugerem que a concepção e a interpretação que o morador faz do serviço de saúde desempenham importante influência no acesso a ele. Essa concepção e interpretação podem se basear na experiência do atendimento

\section{Referências}

1. Andersen RM. Revisiting the behavioral model and access to medical care: does it matter? J Health Soc Behav 1995; 36(1):1-10.

2. Penchansky R, Thomas JW. The concept of access: Definitions and relationship to consumer satisfaction. Med Care 1981; 19(2):127-40.

3. Travassos C, Martins M. Uma revisão sobre os conceitos de acesso e utilização de serviços de saúde. Cad Saude Publica 2004; 20(Supl. 2):190-198.

4. Donabedian A. Aspects of medical care administration. Boston: Harvard University Press; 1973.

5. Santos NR. "SUS - 2010: Espaço para uma Virada". O mundo da saúde 2010;34(1):8-19.

6. Campos GWS, Gutiérrez AC, Guerreiro AVP, Cunha GT. Reflexões sobre a Atenção Básica e a estratégia de Saúde da Família. In: Campos GWS, Guerreiro AVP, organizadores. Manual de práticas de Atenção Básica. São Paulo: Hucitec; 2008. p. 132-153.

7. Santos NR. Política pública de saúde no Brasil: encruzilhada, buscas e escolhas de rumos. Cien Saude Colet 2008; 13(Supl. 2):2009-2018.

8. Brasil. Ministério da Saúde (MS). Conselho Nacional de Saúde. Resolução no 196 de 10 de outubro de 1996. Diretrizes e Normas Regulamentadoras de Pesquisas Envolvendo Seres Humanos. Diário Oficial da União 1996; 16 out.

9. Instituto Brasileiro de Geografia e Estatística (IBGE). [homepage na internet]. Acesso e utilização de serviços de saúde; 2003 [acessado 2011 nov 9]. Disponível em: http://www.ibge.gov.br/home/estatistica/populacao/ trabalhoerendimento/pnad2003/saude/comentario. pdf

10. Corrêa CRS. A avaliação das unidades básicas de saúde segundo os consumidores: a associação entre dados de produção das UBS de Campinas e as avaliações prévia e posterior dos moradores das áreas de cobertura dessas unidades [tese]. Campinas: Universidade Estadual de Campinas; 1998.

11. Oliveira LS, Almeida LGN, Oliveira MAS, Gil GB, Alcione BOC, Medina MG, Pereira RAG. Acessibilidade a atenção básica em um distrito sanitário de Salvador. Cien Saude Colet 2012; 17(11):3047-3056. feito nesse serviço e na classificação que foi feita desse atendimento, mas também podem ter sido fortemente moduladas por aspectos individuais e fatores ligados ao território onde moram.

\section{Colaboradores}

AALM Bonello e CRS Corrêa participaram igualmente de todas as etapas de elaboração do artigo.

12. Halfon N, Wood DL, Valdez RB, Pereyra M, Duan N. Medicaid enrollment and health services access by Latino children in inner-city Los Angeles. JAMA 1997; 277(8):636-641.

13. Newbold KB. Health Care Use and the Canadian Immigrant Population. Int J Health Serv 2009; 39(3):545565 .

14. Flores G, Vega LR. Barriers to Health Care Access for Latino Children: A Review. Fam Med 1998; 30(3):196205.

15. Santos M. O retorno ao Território Apresentação por Maria Adélia Aparecida de Souza. OSAL 2005; (16):251-261.

16. Kontopantelis E, Roland M, Reeves D. Patient experience of access to primary care: identification of predictors in a national patient survey. BMC Family Practice 2010; 11(61):1-15.

17. Travassos C, Oliveira EXG, Viacava F. Desigualdades geográficas e sociais no acesso aos serviços de saúde no Brasil: 1998 e 2003. Cien Saude Colet 2006; 11(4):975986.

18. Barr DA, Wanat SF. Listening to Patients: Cultural and Linguistic Barriers to Health Care Access. Fam Med 2005; 37(3):199-204.

19. Garza-Elizondo ME, Salinas-Martínez AM, Núñez-Rocha G, Villarreal-Ríos E, Moreno-Monsiváis MG. Necesidades de Accesibilidad para Acciones Preventivas. Una Perspectiva de la Población en Monterrey, México en 2005. Rev Esp Salud Pública 2008; 82(5):547-557.

20. Reed RL, Roeger LS, Reinfeld-Kirkman N, Howard SL. Access to general practitioners in South Australia: a population survey. MJA 2008; 189(2):95-99.

Artigo apresentado em 09/08/2013

Aprovado em 28/09/2013

Versão final apresentada em 01/10/2013 